\title{
Closed-Loop System Identification with Recursive Modifications of the Instrumental Variable Method
}

\author{
Nasko ATANASOV ${ }^{1}$, Alexandar ICHTEV ${ }^{2}$ \\ ${ }^{1}$ Department of Automation, Technical University of Varna \\ ${ }^{1}$ Technical University of Varna \\ Studentska 1, 9010 Varna, Bulgaria \\ ${ }^{2}$ Department of Automatics, Technical University of Sofia \\ Kliment Ohridski 8, 1000 Sofia, Bulgaria \\ e-mail:nasko_ratanasov@yahoo.com,ichtev@tu-sofia.bg
}

Received: March 2009; accepted: March 2010

\begin{abstract}
The instrumental variable (IV) method is one of the most renowned methods for parameter estimation. Its bigger advantage is that it is applicable for open-loop as well as for closed-loop systems. The main difficulty in closed-loop identification is due to the correlation between the disturbances and the control signal induced by the loop. In order to overcome this problem, additional excitation signal is introduced. Non-recursive modifications of the instrumental variable method for closed-loop system identification on the base of a generalized IV method have been developed (Atanasov and Ichtev, 2009; Gilson and Van den Hof, 2001; Gilson and Van den Hof, 2003). In this paper, recursive algorithms for theses modifications are proposed and investigated. A simulation is carried out in order to illustrate the obtained results.
\end{abstract}

Keywords: closed-loop system identification, prediction error, instrumental variable method, recursive parameter estimation, observations.

\section{Introduction}

For closed-loop identification, the basic instrumental variable (IV) estimator was first suggested, assuming knowledge of the controller. Further on, it was discussed in details (Vuchkov, 1996; Forsell and Ljung, 1999; Soderstrom et al., 1987). Numbers of nonrecursive computational procedures are also investigated in Atanasov and Ichtev (2009), Gilson and Van den Hof (2001, 2003), Wada et al. (2001).

They are based on modifications of the generalized IV method for closed-loop system identification.

The goal of this paper is to propose recursive modifications to couple of those modifications. The main advantage of the proposed recursive algorithms is their applicability for on-line applications (Pupeikis, 2010; Voros, 2010).

The paper is organized as follows. In the next section the problem statement is presented. In Section 3, the basic and generalized closed-loop IV methods are given. In Section 4, two new recursive algorithms for approximate realizations of the optimal IV 


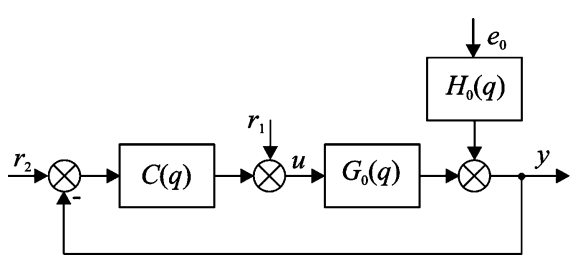

Fig. 1. A closed-loop system to be observed.

estimator are shown. Section 5 presents an example and parameter estimation results. Finally, the conclusions are given in Section 6.

\section{Problem Statement}

Consider a causal, linear, and time-invariant, discrete-time system with output signal $\{y(k)\}$ and plant input signal $\{u(k)\}$, shown in Fig. 1 and expressed by the equations (Atanasov and Ichtev, 2009; Forsell and Ljung, 1999; Gilson and Van den Hof, 2003)

$$
\mid \begin{aligned}
& y(k)=G_{0}(q) u(k)+H_{0}(q) e_{0}(k) \\
& u(k)=r(k)-C(q) y(k)
\end{aligned}
$$

The plant, controller and noise filter are denoted by $G_{0}(q), C(q)$ and $H_{0}(q)$ respectively. Here $k$ is the current number of observations of respective signals, $q$ is time-shift operator such that $q^{-i} u(k)=u(k-i)$ and $\left\{e_{0}(k)\right\}$ is white noise with variance $\sigma_{0}^{2}$. The generalized input signal is introduced as

$$
r(k)=r_{1}(k)+C(q) r_{2}(k)
$$

where the external signals $\left\{r_{2}(k)\right\}$ and $\left\{r_{1}(k)\right\}$ may be regarded as a set point signal and an external excitation signal, which is assumed to be uncorrelated with the noise $\left\{e_{0}(k)\right\}$ (Forsell and Ljung, 1999).

It is assumed that the controller is known and is described in the form

$$
C(q)=\frac{S\left(q^{-1}\right)}{P\left(q^{-1}\right)}=\frac{s_{0}+s_{1} q^{-1}+\cdots+s_{m} q^{-m}}{1+p_{1} q^{-1}+\cdots+p_{m} q^{-m}},
$$

and that the numerator and denominator polynomials of the real plant transfer function

$$
G_{0}(q)=\frac{B_{0}\left(q^{-1}\right)}{A_{0}\left(q^{-1}\right)}
$$

have degree $n_{0}$.

From expressions (1), (3) and (4) equations presented the noise-free and noise parts of the plant output and input signals are obtained:

$$
y(k)=\frac{G_{0}(q)}{1+C(q) G_{0}(q)} r(k)+\frac{H_{0}(q)}{1+C(q) G_{0}(q)} e_{0}(k),
$$




$$
u(k)=\frac{1}{1+C(q) G_{0}(q)} r(k)-\frac{C(q) H_{0}(q)}{1+C(q) G_{0}(q)} e_{0}(k) .
$$

Equation (5) can be written in the form

$$
y(k)=\frac{B_{0}^{\mathrm{cl}}}{A_{0}^{\mathrm{cl}}} r(k)+\frac{1}{A_{0}^{\mathrm{cl}}} \xi(k)
$$

where $\xi(k)=A_{0} P H_{0} e_{0}(k)$ is colour noise generated by $\left\{e_{0}(k)\right\}$ and polynomials $B_{0}^{\text {cl }}$ and $A_{0}^{\text {cl }}$ are from order $\left(n_{0}+m\right)$.

The plant model can be parameterized as

$$
G(q, \theta)=\frac{B\left(q^{-1}, \theta\right)}{A\left(q^{-1}, \theta\right)}=\frac{b_{1} q^{-1}+\cdots+b_{n} q^{-n}}{1+a_{1} q^{-1}+\cdots+a_{n} q^{-n}},
$$

with the parameter vector

$$
\boldsymbol{\theta}=\left[a_{1}, \ldots, a_{n}, b_{1}, \ldots, b_{n}\right]^{T} \in R^{2 n} .
$$

Open-loop $\boldsymbol{f}_{\mathrm{yu}}(k)$ and closed-loop $\boldsymbol{f}_{\mathrm{yr}}(k)$ regressors are defined as

$$
\begin{aligned}
& \boldsymbol{f}_{\mathrm{yu}}(k)=[-y(k-1), \ldots,-y(k-n), u(k-1), \ldots, u(k-n)]^{T}, \\
& \boldsymbol{f}_{\mathrm{yr}}(k)=[-y(k-1), \ldots,-y(k-n-m), r(k-1), \ldots, r(k-l)]^{T},
\end{aligned}
$$

where $l$ is a parameter, chosen by the user.

The following notations are introduced

$$
\begin{aligned}
& \boldsymbol{f}_{r}(k)=[r(k-1), \ldots, r(k-l)]^{T}, \\
& \overline{\boldsymbol{f}}_{\mathrm{yu}}(k)=P\left(q^{-1}\right) \boldsymbol{f}_{\mathrm{yu}}(k), \\
& \bar{y}(k)=P\left(q^{-1}\right) y(k) .
\end{aligned}
$$

The identification is based on data set

$$
Z^{N}=\{r(1), \ldots, r(N), u(1), \ldots, u(N), y(1), \ldots, y(N)\},
$$

which consist of measurements of the generalized input signal $\{r(k)\}$, plant input signal $\{u(k)\}$ and system output $\{y(k)\}$ for $k=1,2, \ldots, N$ (Atanasov and Pupeikis, 2009).

According to indirect approach for closed-loop system identification the closed-loop system (7) is identified and then the elements of vector (9) are determined (Forsell and Ljung, 1999; Pupeikis, 2000). It is possible because the controller $C(q)$ is known.

It could be emphasized that, before the closed-loop indirect parametric identification the respective identifiability conditions should be satisfied according to Atanasov and Ichtev (2009), Vuchkov (1996), Isermann (1984). 
The relation between plant parameters $\boldsymbol{\theta}$ and closed-loop parameters $\boldsymbol{\theta}^{\mathrm{cl}}$ is determined by the following equation

$$
\boldsymbol{\theta}^{\mathrm{cl}}=\boldsymbol{M} \boldsymbol{\theta}+\boldsymbol{\mu}
$$

where the full-column rank matrix $M \in R^{(n+m+l) \times 2 n}$ and the vector $\boldsymbol{\mu} \in R^{n+m+l}$ consist of the coefficients of the controller (Atanasov and Ichtev, 2009; Gilson and Van den Hof, 2001).

The least-squares (LS) estimates $\hat{\boldsymbol{\theta}}_{\mathrm{LS}}^{\mathrm{cl}}$ are obtained by the formula (Atanasov and Ichtev, 2009; Atanasov and Pupeikis, 2009; Daniusis and Vaitkus, 2008)

$$
\hat{\boldsymbol{\theta}}_{\mathrm{LS}}^{\mathrm{cl}}=\left[\sum_{k=1}^{N} \boldsymbol{f}_{\mathrm{yr}}(k) \boldsymbol{f}_{\mathrm{yr}}^{T}(k)\right]^{-1} \sum_{k=1}^{N} \boldsymbol{f}_{\mathrm{yr}}(k) \boldsymbol{y}^{T}(k) .
$$

The parameter vector $\hat{\boldsymbol{\theta}}_{\mathrm{LS}}^{\mathrm{cl}}$ can be estimated by recursive LS method from the form

$$
\begin{aligned}
\hat{\boldsymbol{\theta}}_{\mathrm{LS}}^{\mathrm{cl}}(k)= & \hat{\boldsymbol{\theta}}_{\mathrm{LS}}^{\mathrm{cl}}(k-1)+\frac{\boldsymbol{X}(k-1) f_{\mathrm{yr}}(k)}{1+f_{\mathrm{yr}}^{T}(k) \boldsymbol{X}(k-1) f_{\mathrm{yr}}(k)} \\
& \times\left[y(k)-f_{\mathrm{yr}}^{T}(k) \hat{\boldsymbol{\theta}}_{\mathrm{LS}}^{\mathrm{cl}}(k-1)\right], \\
\boldsymbol{X}(k)= & \boldsymbol{X}(k-1)-\frac{\boldsymbol{X}(k-1) f_{\mathrm{yr}}(k) f_{\mathrm{yr}}^{T}(k) \boldsymbol{X}(k-1)}{1+f_{\mathrm{yr}}^{T}(k) \boldsymbol{X}(k-1) f_{\mathrm{yr}}(k)},
\end{aligned}
$$

with the observation vector $f_{\mathrm{yr}}(k)$ and some initial values of the vector $\hat{\boldsymbol{\theta}}_{\mathrm{LS}}^{\mathrm{cl}}(0)$ and covariance matrix $\boldsymbol{X}(0)$. Here

$$
\hat{\theta}_{\mathrm{LS}}^{\mathrm{cl}}(k)=\left[\hat{a}_{1}^{\mathrm{cl}}, \ldots, \hat{a}_{n+m}^{\mathrm{cl}}, \hat{b}_{1}^{\mathrm{cl}}, \ldots, \hat{b}_{l}^{\mathrm{cl}}\right]^{T} \in R^{n+m+l}
$$

is the current estimate of the vector $\boldsymbol{\theta}^{\mathrm{cl}}=\left[a_{1}, \ldots, a_{n+m}, b_{1}, \ldots, b_{l}\right]^{T} \in R^{n+m+l}$. Since the noise $\{\xi(k)\}$ is colour the LS estimates $\hat{\boldsymbol{\theta}}_{\mathrm{LS}}^{\mathrm{cl}}$ are inconsistent and biased. This drawback is not present in the IV method and thus this is one applicable solution.

\section{Basic and Generalized Closed-Loop IV Methods}

The basic closed-loop IV method utilizes $2 n$ time-shifted values of the reference signal as instruments. Thus, the estimates are calculated according to the relation (Soderstrom et al., 1987)

$$
\hat{\boldsymbol{\theta}}_{\mathrm{IV}}=\left[\sum_{k=1}^{N} \boldsymbol{z}(k) \boldsymbol{f}_{\mathrm{yu}}^{T}(k)\right]^{-1} \sum_{k=1}^{N} \boldsymbol{z}(k) \boldsymbol{y}^{T}(k),
$$

where $z(k)=\boldsymbol{f}_{r}(k)$ and $l=2 n$. 
The parameter vector $\boldsymbol{\theta}$ can be estimated by recursive IV method from the form

$$
\begin{aligned}
\hat{\boldsymbol{\theta}}_{\mathrm{IV}}(k)= & \hat{\boldsymbol{\theta}}_{\mathrm{IV}}(k-1)+\frac{\boldsymbol{E}(k-1) \boldsymbol{z}(k)}{1+f_{\mathrm{yu}}^{T}(k) \boldsymbol{E}(k-1) \boldsymbol{z}(k)} \\
& \times\left[y(k)-f_{\mathrm{yu}}^{T}(k) \hat{\boldsymbol{\theta}}_{\mathrm{IV}}(k-1)\right], \\
\boldsymbol{E}(k)= & \boldsymbol{E}(k-1)-\frac{\boldsymbol{E}(k-1) \boldsymbol{z}(k) f_{\mathrm{yu}}^{T}(k) \boldsymbol{E}(k-1)}{1+f_{\mathrm{yu}}^{T}(k) \boldsymbol{E}(k-1) \boldsymbol{z}(k)},
\end{aligned}
$$

with the vector of observations $f_{\mathrm{yu}}(k)$ and some initial values of the vector $\hat{\boldsymbol{\theta}}_{\mathrm{IV}}(0)$ and covariance matrix $\boldsymbol{E}(0)$. Here

$$
\hat{\theta}_{\mathrm{IV}}(k)=\left[\hat{a}_{1}, \ldots, \hat{a}_{n}, \hat{b}_{1}, \ldots, \hat{b}_{n}\right]^{T} \in R^{2 n}
$$

is the current estimate of the vector $\boldsymbol{\theta}=\left[a_{1}, \ldots, a_{n}, b_{1}, \ldots, b_{n}\right]^{T} \in R^{2 n}$.

The quality of the IV estimates is increased by the generalized closed-loop IV method. The obtained estimates are generalization of the basic IV ones. This is achieved by filtering input-output data and by gaining advantage from the augmented instruments $z(k) \in R^{n_{z}}$, where $n_{z} \geqslant 2 n$. In this case the estimates are obtained by the formula

$$
\begin{aligned}
\hat{\boldsymbol{\theta}}_{\mathrm{IV}}^{G}(N)= & \arg \underbrace{\min }_{\boldsymbol{\theta}} \|\left[\frac{1}{N} \sum_{k=1}^{N} \boldsymbol{z}(k) L\left(q^{-1}\right) \boldsymbol{f}_{\mathrm{yu}}^{T}(k)\right] \boldsymbol{\theta} \\
& -\left[\frac{1}{N} \sum_{k=1}^{N} \boldsymbol{z}(k) L\left(q^{-1}\right) y(k)\right] \|_{\boldsymbol{Q}}^{2}
\end{aligned}
$$

where $L\left(q^{-1}\right)$ is a stable filter and $\|\boldsymbol{x}\|_{\boldsymbol{Q}}^{2}$ is a quadratic form $\boldsymbol{x}^{T} \boldsymbol{Q} \boldsymbol{x}$ with a positive definite weighting matrix $\boldsymbol{Q}$ (Gilson and Van den Hof, 2003).

The estimated accuracy is characterized by the variances and covariances of the estimates, which are provided from the covariance matrix. The covariance matrix is dependant on the choice of the instruments $z(k)$ from dimension $n_{z}$, weighting matrix $\boldsymbol{Q}$ and stable filter $L\left(q^{-1}\right)$. Then the optimal covariance matrix $\boldsymbol{P}_{\mathrm{IV}}^{\text {opt }}$ is obtained for the closed-loop identification (Atanasov and Ichtev, 2009; Gilson and Van den Hof, 2003) if

$$
\begin{aligned}
& \boldsymbol{z}(k)=\frac{1}{\sigma_{0}^{2}}\left\{\left[A_{0}\left(q^{-1}\right) H_{0}\left(q^{-1}\right)\right]^{-1} \tilde{f}_{\mathrm{yu}}^{T}(k)\right\}^{T}, \\
& n_{z}=2 n, \\
& \boldsymbol{Q}=\boldsymbol{I}, \\
& L\left(q^{-1}\right)=\left[A_{0}\left(q^{-1}\right) H_{0}\left(q^{-1}\right)\right]^{-1} .
\end{aligned}
$$

Here $\tilde{f}_{\mathrm{yu}}^{T}(k)$ denotes the noise-free part of $f_{\mathrm{yu}}(k)$. The optimal accuracy cannot be achieved in practice. This is evident from the last equation (29), from where it can be concluded that the optimal IV estimator is only feasible if the true plant and noise models are exactly known. 


\section{Recursive Algorithms for Approximate Realizations of the Optimal Closed-Loop IV Estimator}

In order to create an approximate realization of the optimal closed-loop IV estimator there are two main requirements. In first place, for the determination of the filter $L\left(q^{-1}\right)$ and the instruments $\boldsymbol{z}(k)$ it is important the choice of the noise model. The other requirement is regarding the choice of the initial plant model. The second condition is important for the computation of the noise-free part of the regressor $f_{\mathrm{yu}}(k)$.

\subsection{Recursive Algorithm for Extension to the IV4 Method}

An approximation of the optimal closed-loop IV method can be obtained, by extending IV4 method for open-loop identification (Gilson and Van den Hof, 2003). Utilization of instruments that are uncorrelated with the noise part of the plant input signal, but correlated with the noise-free part is the keystone principle.

For the model structure presented as an linear regression model

$$
\hat{y}(k, \theta)=f_{\mathrm{yu}}^{T}(k) \boldsymbol{\theta}
$$

the estimation is based on the following recursive algorithm:

1. The parameter vector $\boldsymbol{\theta}$ is estimated by recursive LS method from the form

$$
\begin{aligned}
& \hat{\boldsymbol{\theta}}_{\mathrm{LS}}^{1}(k)= \hat{\boldsymbol{\theta}}_{\mathrm{LS}}^{1}(k-1)+\frac{\boldsymbol{\Gamma}(k-1) f_{\mathrm{yu}}(k)}{1+f_{\mathrm{yu}}^{T}(k) \boldsymbol{\Gamma}(k-1) f_{\mathrm{yu}}(k)} \\
& \times\left[y(k)-f_{\mathrm{yu}}^{T}(k) \hat{\boldsymbol{\theta}}_{\mathrm{LS}}^{1}(k-1)\right], \\
& \boldsymbol{\Gamma}(k)=\boldsymbol{\Gamma}(k-1)-\frac{\boldsymbol{\Gamma}(k-1) f_{\mathrm{yu}}(k) f_{\mathrm{yu}}^{T}(k) \boldsymbol{\Gamma}(k-1)}{1+f_{\mathrm{yu}}^{T}(k) \boldsymbol{\Gamma}(k-1) f_{\mathrm{yu}}(k)},
\end{aligned}
$$

with the vector of observations $f_{\mathrm{yu}}(k)$ and some initial values of the vector $\hat{\boldsymbol{\theta}}_{\mathrm{LS}}^{1}(0)$ and covariance matrix $\boldsymbol{\Gamma}(0)$. Here

$$
\hat{\theta}_{\mathrm{LS}}^{1}(k)=\left[\hat{a}_{1}^{1}, \ldots, \hat{a}_{n}^{1}, \hat{b}_{1}^{1}, \ldots, \hat{b}_{n}^{1}\right]^{T} \in R^{2 n}
$$

is the current estimate of the vector $\boldsymbol{\theta}=\left[a_{1}, \ldots, a_{n}, b_{1}, \ldots, b_{n}\right]^{T} \in R^{2 n}$. The corresponding transfer function $\hat{G}_{1 k}(q)$ is formed by the elements of this estimate.

2. Generate current instruments $\boldsymbol{z}_{1}(k)$ as estimates of the noise free part of the openloop regressor $f_{\mathrm{yu}}(k)$ by relations

$$
\begin{aligned}
& \tilde{y}_{1}(k)=\frac{\hat{G}_{1 k}(q)}{1+C(q) \hat{G}_{1 k}(q)} r(k), \\
& \tilde{u}_{1}(k)=\frac{1}{1+C(q) \hat{G}_{1 k}(q)} r(k), \\
& \boldsymbol{z}_{1}(k)=\left[-\tilde{y}_{1}(k-1), \ldots,-\tilde{y}_{1}(k-n) \tilde{u}_{1}(k-1), \ldots, \tilde{u}_{1}(k-n)\right]^{T} .
\end{aligned}
$$


3. The parameter vector $\boldsymbol{\theta}$ is estimated by recursive IV method from the form

$$
\begin{aligned}
& \hat{\boldsymbol{\theta}}_{\mathrm{IV}}^{2}(k)= \hat{\boldsymbol{\theta}}_{\mathrm{IV}}^{2}(k-1)+\frac{\boldsymbol{\Lambda}(k-1) \boldsymbol{z}_{1}(k)}{1+f_{\mathrm{yu}}^{T}(k) \boldsymbol{\Lambda}(k-1) \boldsymbol{z}_{1}(k)} \\
& \times\left[y(k)-f_{\mathrm{yu}}^{T}(k) \hat{\boldsymbol{\theta}}_{\mathrm{IV}}^{2}(k-1)\right], \\
& \boldsymbol{\Lambda}(k)=\boldsymbol{\Lambda}(k-1)-\frac{\boldsymbol{\Lambda}(k-1) \boldsymbol{z}_{1}(k) f_{\mathrm{yu}}^{T}(k) \boldsymbol{\Lambda}(k-1)}{1+f_{\mathrm{yu}}^{T}(k) \boldsymbol{\Lambda}(k-1) \boldsymbol{z}_{1}(k)},
\end{aligned}
$$

with the vector of observations $f_{\mathrm{yu}}(k)$ and some initial values of the vector $\hat{\boldsymbol{\theta}}_{\mathrm{IV}}^{2}(0)$ and covariance matrix $\boldsymbol{\Lambda}(0)$. The corresponding estimated $n$-order transfer function is $\hat{G}_{2 k}(q)=\frac{\hat{B}_{2 k}\left(q^{-1}\right)}{\hat{A}_{2 k}\left(q^{-1}\right)}$.

4. Calculate the prediction error of the $k$-th iteration

$$
\hat{\rho}(k)=\hat{A}_{2 k}\left(q^{-1}\right) y(k)-\hat{B}_{2 k}\left(q^{-1}\right) u(k),
$$

and generate $2 n$-order prediction error vector $\hat{\boldsymbol{w}}$ as

$$
\hat{\boldsymbol{w}}(k)=[\hat{\rho}(k-1), \hat{\rho}(k-2), \ldots, \hat{\rho}(k-2 n)]^{T} .
$$

5. By recursive LS method, with some initial values of the vector $\hat{l}(0)$ and covariance matrix $\boldsymbol{\Psi}(0)$, calculate the current elements of the filter with equation $L\left(q^{-1}\right)=1+$ $l_{1} q^{-1}+l_{2} q^{-2}+\cdots+l_{2 n} q^{-2 n}$, that convert $\{\hat{\rho}(k)\}$ into white noise $\{e(k)\}$

$$
\begin{aligned}
\hat{l}(k)= & \hat{l}(k-1)+\frac{\boldsymbol{\Psi}(k-1) \hat{w}(k)}{1+\hat{w}^{T}(k) \boldsymbol{\Psi}(k-1) \hat{w}(k)} \\
& \times[e(k)-\hat{w}(k) \hat{l}(k-1)], \\
\boldsymbol{\Psi}(k)= & \boldsymbol{\Psi}(k-1)-\frac{\boldsymbol{\Psi}(k-1) \hat{\boldsymbol{w}}(k) \hat{\boldsymbol{w}}^{T}(k) \boldsymbol{\Psi}(k-1)}{1+\hat{\boldsymbol{w}}^{T}(k) \boldsymbol{\Psi}(k-1) \hat{\boldsymbol{w}}(k)} .
\end{aligned}
$$

6. Form the current filter

$$
\hat{L}_{k}\left(q^{-1}\right)=1+\hat{l}_{1}(k) q^{-1}+\hat{l}_{2}(k) q^{-2}+\cdots+\hat{l}_{2 n}(k) q^{-2 n}
$$

by the estimate $\hat{l}(k)=\left[\hat{l}_{1}(k), \hat{l}_{2}(k), \ldots, \hat{l}_{2 n}(k)\right]^{T}$.

7. Generate current instruments $\boldsymbol{z}_{2}(k)$ as

$$
\begin{aligned}
\tilde{y}_{2}(k) & =\frac{\hat{G}_{2}(q)}{1+C(q) \hat{G}_{2}(q)} r(k), \\
\tilde{u}_{2}(k) & =\frac{1}{1+C(q) \hat{G}_{2}(q)} r(k), \\
\boldsymbol{z}_{2}(k) & =\left[-\tilde{y}_{2}(k-1), \ldots,-\tilde{y}_{2}(k-n), \tilde{u}_{2}(k-1), \ldots, \tilde{u}_{2}(k-n)\right]^{T} .
\end{aligned}
$$


8. Filter the input-output data

$$
\boldsymbol{f}_{\mathrm{yu} F}(k)=\hat{L}_{k}\left(q^{-1}\right) \boldsymbol{f}_{\mathrm{yu}}(k), \quad y_{F}(k)=\hat{L}_{k}\left(q^{-1}\right) y(k) .
$$

9. Recursive determine the IV4 estimates as

$$
\begin{aligned}
\hat{\boldsymbol{\theta}}_{\mathrm{IV} 4}(k)= & \hat{\boldsymbol{\theta}}_{\mathrm{IV} 4}(k-1)+\frac{\boldsymbol{\Sigma}(k-1) \boldsymbol{z}_{1}(k)}{1+f_{\mathrm{yu} F}^{T}(k) \boldsymbol{\Sigma}(k-1) \boldsymbol{z}_{1}(k)} \\
& \times\left[y_{F}(k)-f_{\mathrm{yu} F}^{T}(k) \hat{\boldsymbol{\theta}}_{\mathrm{IV} 4}(k-1)\right] \\
\boldsymbol{\Sigma}(k)= & \boldsymbol{\Sigma}(k-1)-\frac{\boldsymbol{\Sigma}(k-1) \boldsymbol{z}_{2}(k) f_{\mathrm{yu} F}^{T}(k) \boldsymbol{\Sigma}(k-1)}{1+f_{\mathrm{yu} F}^{T}(k) \boldsymbol{\Sigma}(k-1) \boldsymbol{z}_{2}(k)},
\end{aligned}
$$

with the vector of observations $f_{\mathrm{yu} F}(k)$ and some initial values of the vector $\hat{\boldsymbol{\theta}}_{\mathrm{IV} 4}(0)$ and covariance matrix $\boldsymbol{\Sigma}(0)$.

\subsection{Recursive Algorithm for Closed-Loop Quasi-Optimal IV Method}

One way to initialize the estimation procedure is by estimation of the noise and plants models with high-order LS estimator $\left(n_{1}>n\right)$. The biased estimates obtained by this approach are only in the first step of the algorithm and does not lead to a bias in the final model (Gilson and Van den Hof, 2003).

The estimation is based on the following recursive algorithm:

1. Present the ARX model structure as a linear regression model by (30) and estimate $\boldsymbol{\theta}$ by a high-order recursive LS estimator (31) and (32) with the vector of observation

$$
\begin{aligned}
\boldsymbol{f}_{\text {hyu }}(k)= & {\left[-y(k-1), \ldots,-y(k-n), \ldots,-y\left(k-n_{1}\right), u(k-1), \ldots,\right.} \\
& \left.u(k-n), \ldots, u\left(k-n_{1}\right)\right]^{T} .
\end{aligned}
$$

Obtain a current estimate $\hat{\boldsymbol{\theta}}_{\mathrm{LS}}^{1}(k)$ along with the plant and noise models

$$
\hat{G}_{1}(q)=\frac{\hat{B}_{1}\left(q^{-1}\right)}{\hat{A}_{1}\left(q^{-1}\right)} ; \quad \hat{H}_{1}(q)=\frac{1}{\hat{A}_{1}\left(q^{-1}\right)} .
$$

2. In this case the filter is given by

$$
\hat{L}\left(q^{-1}\right)=\left[\hat{A}_{1}\left(q^{-1}\right) \hat{H}_{1}\left(q^{-1}\right)\right]^{-1}=1
$$

3. Compute the current noise-free part $\tilde{f}_{\text {yu }}(k)$ of the open-loop regressors

$$
\tilde{f}_{\mathrm{yu}}(k)=\left[-\tilde{y}_{1}(k-1), \ldots,-\tilde{y}_{1}(k-n), \tilde{u}_{1}(k-1), \ldots, \tilde{u}_{1}(k-n)\right]^{T},
$$

with $\tilde{y}_{1}(k)$ and $\tilde{u}_{1}(k)$, computed according to (34) and (35). 
4. Generate the current instruments as

$$
\boldsymbol{z}(k)=\left\{\left[A_{1}\left(q^{-1}\right) H_{1}\left(q^{-1}\right)\right]^{-1} \tilde{f}_{\mathrm{yu}}^{T}(k)\right\}^{T} \equiv \tilde{f}_{\mathrm{yu}}(k) .
$$

5. Determine the quasi-optimal recursive IV estimates of $\boldsymbol{\theta}$ as

$$
\begin{aligned}
& \hat{\boldsymbol{\theta}}_{\mathrm{IV}}^{\mathrm{OPT}}(k)= \hat{\boldsymbol{\theta}}_{\mathrm{IV}}^{\mathrm{OPT}}(k-1)+\frac{\boldsymbol{\Omega}(k-1) \mathbf{z}(k)}{1+f_{\mathrm{yu}}^{T}(k) \boldsymbol{\Omega}(k-1) \mathbf{z}(k)} \\
& \times\left[y(k)-f_{\mathrm{yu}}^{T}(k) \hat{\boldsymbol{\theta}}_{\mathrm{IV}}^{\mathrm{OPT}}(k-1)\right] \\
& \boldsymbol{\Omega}(k)=\boldsymbol{\Omega}(k-1)-\frac{\boldsymbol{\Omega}(k-1) \mathbf{z}(k) f_{\mathrm{yu}}^{T}(k) \boldsymbol{\Omega}(k-1)}{1+f_{\mathrm{yu}}^{T}(k) \boldsymbol{\Omega}(k-1) \mathbf{z}(k)}
\end{aligned}
$$

with the vector of observations $f_{\mathrm{yu}}(k)$ and some initial values of the vector $\hat{\boldsymbol{\theta}}_{\mathrm{IV}}^{\mathrm{OPT}}(0)$ and covariance matrix $\boldsymbol{\Omega}(0)$.

\section{Example}

Consider a close-loop system, described in the form of (2), (5) and (6) with

$$
\begin{aligned}
& G_{0}(q)=\frac{0.75 q^{-1}}{1-0.385 q^{-1}} \\
& C(q)=\frac{0.1387 q^{-1}+0.0889 q^{-2}}{1-1.036 q^{-1}+0.2636 q^{-2}} \\
& H_{0}(q)=\frac{1+0.05 q^{-1}+0.8 q^{-2}}{1-1.036 q^{-1}+0.2636 q^{-2}}
\end{aligned}
$$

The input $-\{r(k)\}$ and the noise $-\left\{e_{0}(k)\right\}$ are random signals with normal distribution. The standard deviation of the generalized input signal $\{r(k)\}$ is one. The desired noise to signal ratio is obtained by variation of the standard deviation of the noise $\left\{e_{0}(k)\right\}$.

The relative mean-squared error $Q_{\theta}$ with respect to the true parameters is used as an accuracy criterion. The plant parameters are recursively estimated on the basis of the data set (15). Simulations have been performed for three different noise to signal ratios $\mathrm{rel}_{1}=1 \% \mathrm{rel}_{2}=5 \%$ and $\mathrm{rel}_{3}=10 \%$.

Two estimators are investigated - recursive extended IV4 and recursive quasi-optimal IV methods. For comparison purposes, the recursive optimal IV estimator is used. This benchmark is obtained according to (25), (26)-(29) and the true plant and noise filter parameters from (57) and (59).

The true plant parameters are $a_{1}=-0.385$ and $b_{1}=0.75$. The values of the parameter estimates after 1000 iterations and the values of the relative mean-squared errors $Q_{\theta}$ (on the last iteration) with respect to the true parameters are presented in Table 1. 
Table 1

Parameter estimation results

\begin{tabular}{lrrrrrr}
\hline & rel $_{1}=1 \%$ & rel $_{2}=5 \%$ & \multicolumn{3}{c}{ rel $_{3}=10 \%$} \\
\hline$\hat{\boldsymbol{\theta}}_{\text {IV }}^{\text {Bencmark }}$ & -0.3853 & 0.7508 & -0.3839 & 0.7559 & -0.3827 & 0.7567 \\
$Q_{\theta}$ & 0.0010 & & 0.0071 & & 0.0084 & \\
$\hat{\boldsymbol{\theta}}_{\text {IV4 }}$ & -0.3791 & 0.7533 & -0.3760 & 0.7668 & -0.4146 & 0.7183 \\
$Q_{\theta}$ & 0.0080 & & 0.0226 & & 0.0514 & \\
$\hat{\boldsymbol{\theta}}_{\text {IV }}^{\text {OPT }}$ & -0.3845 & 0.7499 & -0.3862 & 0.7478 & -0.4256 & 0.7258 \\
$Q_{\theta}$ & 0.0006 & & 0.0030 & & 0.0561 & \\
\hline
\end{tabular}

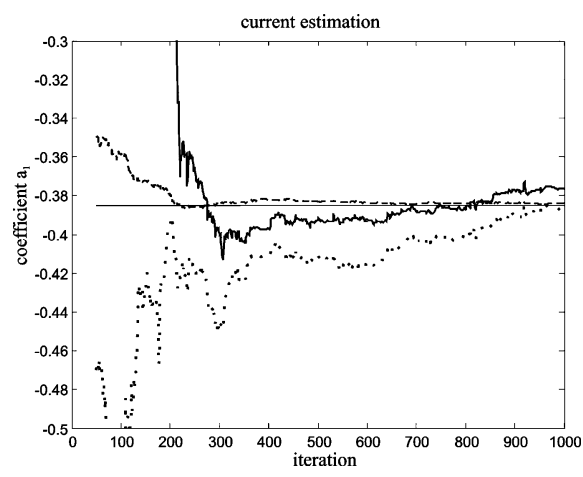

Fig. 2a. Estimations for $\operatorname{rel}_{1}=1 \%$

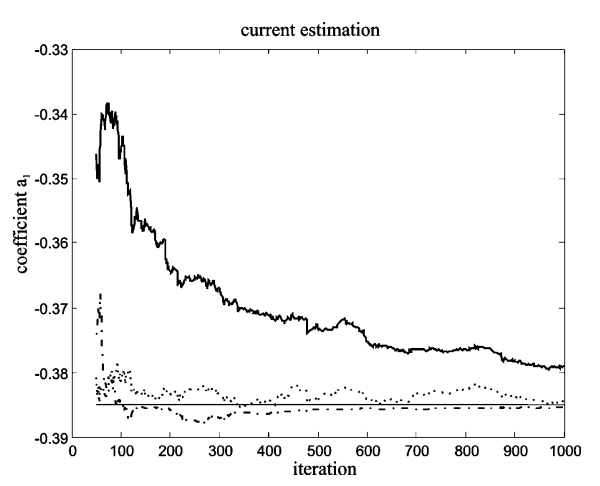

Fig. 3a. Estimations for $\operatorname{rel}_{1}=5 \%$

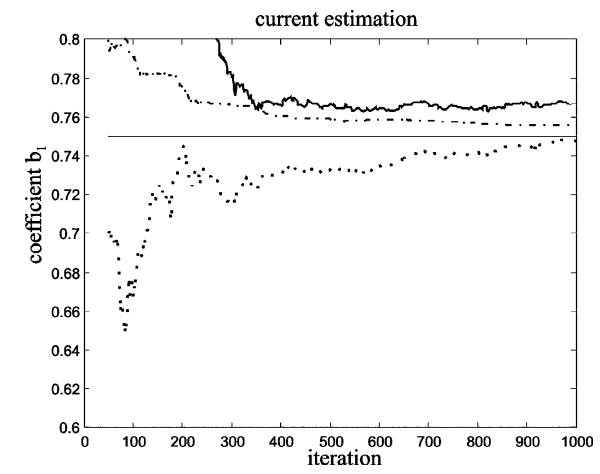

Fig. 2b. Estimations for $\mathrm{rel}_{2}=1 \%$.

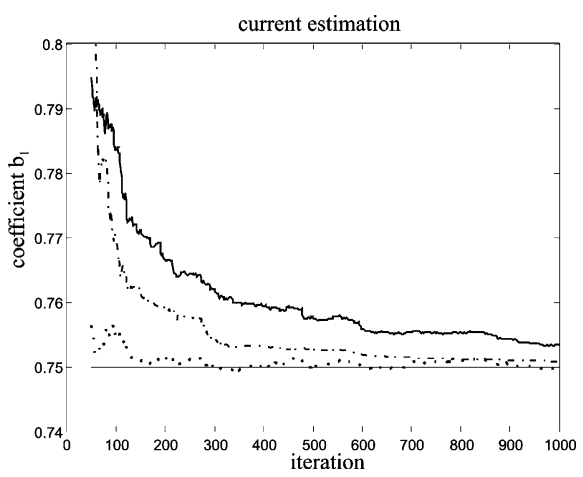

Fig. 3b. Estimations for rel $_{2}=5 \%$.

The parameter estimation results are illustrated in Figs. 2, 3 and 4. The benchmark estimates are marked by dashdot line, the extended IV 4 - by solid line, and the quasioptimal IV - by dotted line. 


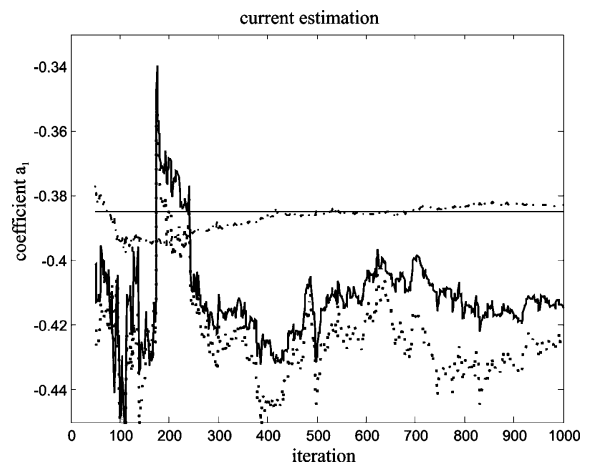

Fig. 4a. Estimations for $\mathrm{rel}_{3}=10 \%$

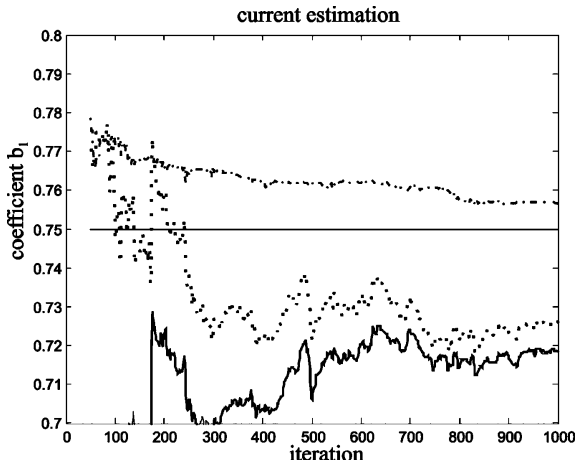

Fig. 4b. Estimations for $\mathrm{rel}_{3}=10 \%$.

\section{Conclusion}

In this paper two new recursive IV estimators are proposed. They approximate the optimal closed-loop IV estimator that is used as benchmark.

An experiment is carried out. It can be noticed that after substantial initial oscillations, up to the 50th iteration (not shown on the figures), the estimates, obtained from the recursive estimators, converge towards their true values. From Table 1, Fig. 2, 3 and 4 it can also be seen that with respect to the convergence, the best estimator is the recursive standard optimal IV estimator (dashdot line). Unfortunately, this benchmark cannot be implemented in practise. The second best is the recursive quasi-optimal IV estimator (dotted line), which algorithm incorporates intermediate high order LS estimates. Moreover, in the case of small noise to signal ratio it can provide more accurate estimates. The initial LS estimates and approximate determination of the noise free part of the openloop repressors have negative impact on the convergence and accuracy of the extended IV4 and quasi-optimal IV estimators, especially in the case of high noise to signal ratio.

For future investigation the authors are planning to develop robust recursive versions of the investigated algorithms in order to perform identification in real time in the presence of outliers in observations.

\section{References}

Atanasov, N., Ichtev, A. (2009). Closed-loop system identification with modifications of the instrumental variable method. Informatica, 21(2), 159-174.

Atanasov, N., Pupeikis, R. (2009). On recursive calculation of M- and GM-estimates by direct identification in LQG control systems. Informatica, 20(1), 3-22.

Vuchkov, I. (1996). Identification. Eurapel, Sofia (in Bulgarian).

Daniusis, P., Vaitkus, P. (2008). Neural network with matrix inputs. Informatica, 19(4), 477-486.

Forsell, U., Ljung, L. (1999). Closed-loop identification revisited. Automatica, 35(7), 1215-1241.

Gilson, M., Van den Hof, P. (2001). On the relation between a bias-eliminated least squares BELS and an IV estimator in closed-loop identification. Automatica, 37(10), 1593-1600.

Gilson, M., Van den Hof, P. (2003). IV methods for closed-loop system identification. In: 13th IFAC Symposium on System Identification, pp. 537-542. 
Isermann, R. (1984). Digital Control System. Springer/Mir, Moskow/Berlin (in Russian).

Pupeikis, R. (2000). Closed-loop robust identification using the indirect approach. Informatica, 11(3), 297-310.

Pupeikis, R. (2010). On a time-varying parameter adaptive self-organizing system in the presence of large outliers in observations. Informatica, 21(1), 79-94.

Soderstrom, T., Stoica, P., Trulsson, E. (1987). Instrumental variable methods for closed-loop systems, In: 10th IFAC World Congress, Munich, Germany, pp. 363-368.

Voros, J. (2010). Recursive identification of systems with noninvertible output nonlinearities. Informatica, 21(1), 139-148.

Wada, K., Jia, L., Hanada, T., Imai, J. (2001). On BELS parameter estimation method of transfer functions. Transactions of the Institute of Electrical Engineers of Japan, 121-C, 795-799.

N. Atanasov, $\mathrm{PhD}$, is associated professor at Department of Automation, Technical University of Varna. His main research interests are in robust identification, robust estimation, control theory, adaptive control.

A. Ichtev, PhD, is associated professor at Department of Automatics, Systems and Control Group, Technical University of Sofia. His main research interests are in control theory, adaptive control, fuzzy systems, robust control, fault detection, fault tolerant control.

\section{Grižtamojo ryšio sistemos identifikavimas, taikant instrumentiniu kintamuju metodo rekurentines modifikacijas}

\section{Nasko ATANASOV, Alexandar ICHTEV}

Šiame straipsnyje pateikiama keletas apibendrinto instrumentinių kintamujų (IK) metodo rekurentiniu modifikaciju grị̌tamojo ryšio sistemos identifikavimui. Pasiūlyti du nauji rekurentiniai algoritmai, tam tikra prasme aproksimuojantys optimalaus IK metodo ịverčiu realizacijas. Pagrindinis gautų rekurentinių išraišku privalumas tas, kad jos esti nesudètingos bei patogios realizuoti praktiškai. Pateiktas grị̌tamojo ryšio sistemos pavyzdys ir gauti modeliavimo kompiuteriu rezultatai. 